\section{Behavioral contrast and inhibitive stimulus control ${ }^{1}$}

MATTHEW YARCZOWER, Bryn Mawr College, Bryn Mawr, Pa. 19010

Inhibitive stimulus control was present early in discriminative training but weakened with continued exposure to the same discriminative training procedures. Presence and absence of inhibitive stimulus control appeared to be related to the presence or absence of behavioral contrast.

A technique for the measurement of gradients of inhibitive effects was introduced by Honig et al (1963). This procedure involves discriminative training with $\mathrm{S}_{+}$and $\mathrm{S}_{-}$, in which $\mathrm{S}_{-}$is on a different dimension than is $\mathrm{St}$. For example, $\mathrm{S}+$ might be a 550 -nm key light, and $S$ - might be a white, vertical line that appears on the response key. Clearly, both stimuli have some common features; they are presented on the same response key and as visual stimuli are on the brightness dimension. However, changes in the angular orientation of the white line does not alter its similarity to the wavelength dimension. Thus, the two dimensions are said to be orthogonal or independent of one another. Inhibitive stimulus control is evident in generalization testing when responses are more frequently emitted as the stimulus becomes more remote from the $\mathrm{S}-$.

There are indications that inhibitive stimulus control may be related to certain performance changes that occur during discriminative training (Terrace, 1966). Pigeons were given discriminative training that consisted of interspersed presentations of wavelength stimuli, a VI 1-min schedule of positive reinforcement in the presence of one wavelength and nonreinforcement in the presence of the other. The discriminative training schedule resulted in a rate increase in the presence of S+ early in training. Rate of responding returned to its prediscriminative training level with continued training. Generalization tests

Fig. 1. Response rates and generalization gradients are shown for individual pigeons tested while rates in the presence of $S+$ were higher than they were prior to discriminative training. The dashed line in the section in which $\mathbf{S}+$ and $S$ - rates are shown is the mean response rate of the 5 days prior to discriminative training. The filled circles are data obtained when the $S-$ was "superimposed" on the St. The open circles are data gathered without "superimposition." conducted early in training yielded a shift in the stimulus associated with modal responding ("peak shift") and a marked asymmetry in the distribution of responses in the presence of test stimuli. Generalization tests carried out after extensive discriminative training showed less of a shift in the modal responding and less of the asymmetry (see Fig. 1, Terrace, 1966), contrary to what would be expected if inhibitive stimulus control was unchanged.

The present experiment has a twofold purpose. One is to assess the relation between certain rate changes during discriminative training and inhibitive stimulus control; the second purpose is to compare two methods of testing for inhibitive stimulus control when the stimuli used in discriminative training are on different dimensions.

\section{SUBJECTS AND APPARATUS}

Eight experimentally naive Silver King pigeons, 3-4 years old, were maintained at $80 \%$ of their free-feeding body weights throughout the course of the experiment. The experimental chamber was made of Plexiglas and measured $35.6 \times 35.6 \times 38.1 \mathrm{~cm}$. The response panel consisted of a standard Gerbrands pigeon key that was made of clear Plexiglas and behind which was a projector made by Industrial Electronic Engineers Co., Model No. 10-1279-1829L, which could project any one of nine white line tilts and

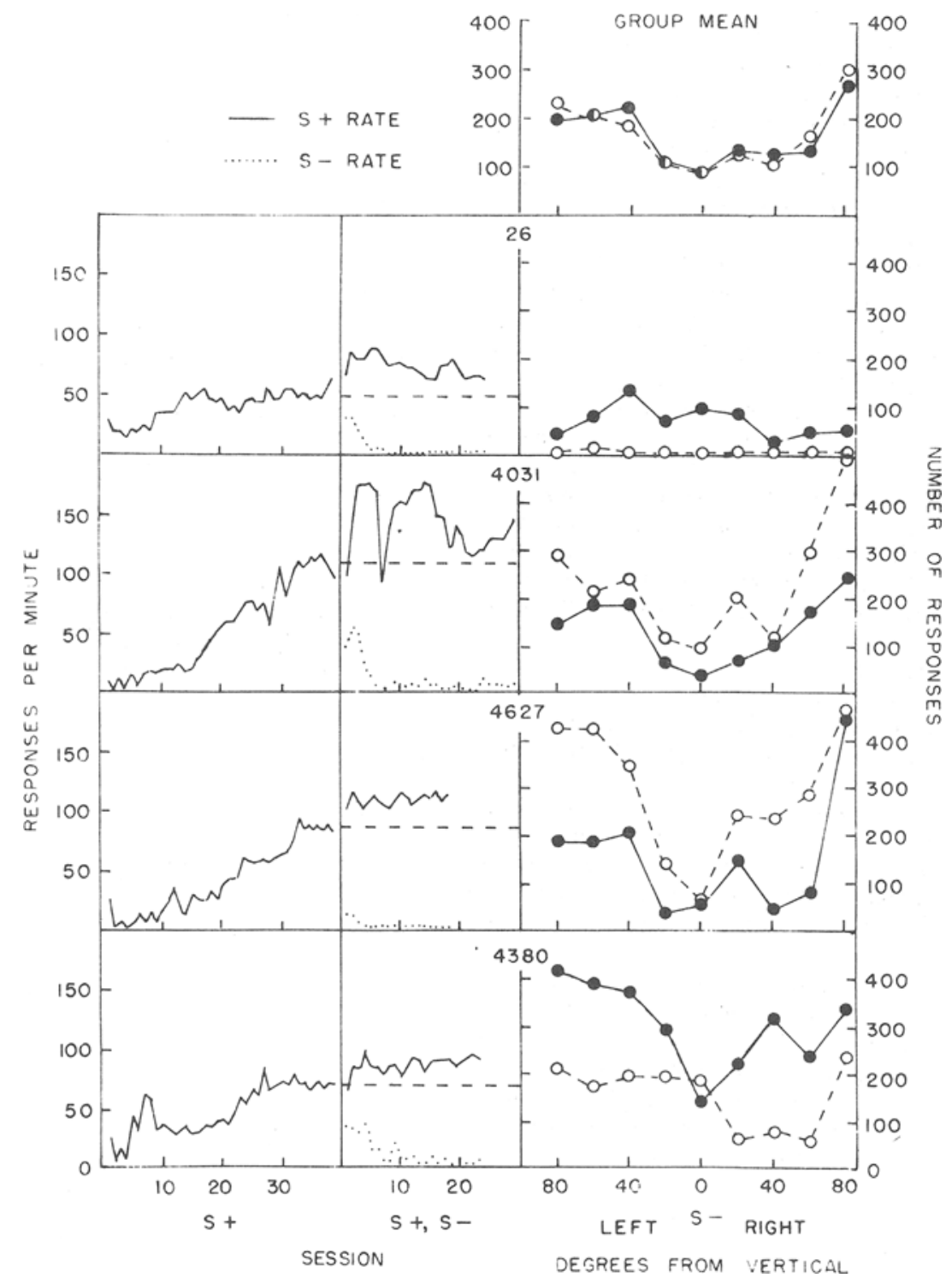


any one of three wavelengths. A Lehigh Valley Electronics Co. feeder was located below the response key.

\section{PROCEDURE}

All pigeons were magazine-trained on the 1st day and given 25 reinforcements on each of 2 days in which each key peck in the presence of a 550-nm key light was followed by access to the food magazine for $3 \mathrm{sec}$. After this training, the schedule of reinforcement was changed to a variable interval schedule in which the mean intervai was $1 \mathrm{~min}$ (VI 1 -min). These training sessions consisted of $201-\mathrm{min}$ presentations of the $550-\mathrm{nm}$ light. Each trial was separated from the next by a $2-\mathrm{sec}$ period of time during which all lights were turned off and no reinforcements were available, i.e., a time-out (TO).

Discriminative training began on the 40th session of the variable-interval schedule of reinforcement. The VI 1-min schedule in the presence of the 550-nm light ( $\mathrm{S}+$ ) was alternated with a condition during which nonreinforcement of key pecks was in effect in the presence of a white vertical line $(\mathrm{S}-$ ) that was $5 \mathrm{~mm}$ wide. The background of the key was black. The duration of each stimulus period was $1 \mathrm{~min}$, with a $2-\mathrm{sec}$ TO between stimulus presentations. The 20 presentations of St and the 20 presentations of $\mathrm{S}-$ followed Gellermann series (1933).

A rate increase in the presence of St results when discriminative training is introduced. Half of the birds were given generalization tests while their response rates were still higher than they were before discriminative training, and half were given generalization tests after their response rates had returned to the level that existed prior to discriminative training.

Generalization tests were conducted on each of 4 days. Each day began with four trials in which the same discriminative training schedule was in effect. Generalization tests were carried out with nonreinforcement and consisted of six presentations of each of nine lines of varying orientation ( $10 \mathrm{deg}$ to $170 \mathrm{deg}$, in 20-deg steps), each for $1 \mathrm{~min}$, with $2 \mathrm{sec}$ of

Fig. 2. Response rates and generalization gradients are shown for individual pigeons tested when rates in the presence of $S+$ had returned to the level prior to discriminative training. The dashed line in the section in which $S+$ and $S-$ rates are shown is the mean response rate of the 5 days prior to discriminative training. The filled circles are data obtained when the $S-$ was "superimposed" on the St. The open circles are data gathered without "superimposition." darkness between each test trial. All birds received tests with white lines against a black background on Day 1 and on Day 4 . On Days 2 and 3, the line stimulus was presented against a background of the same wavelength as the S+ during training.

\section{RESULTS}

When discriminative training was introduced, response rate in $\mathrm{S}+$ increased for all birds. Figure 1 depicts the training data and the generalization gradients of birds that were tested when response rates were still higher than they were prior to discriminative training. There are two sets of test data shown for each bird, one in which the test line was projected on a black background and one in which the background was of the same wavelength as St.

The data of three of the four birds clearly indicate inhibitive stimulus controlcontrol- and the other is that there was a greater

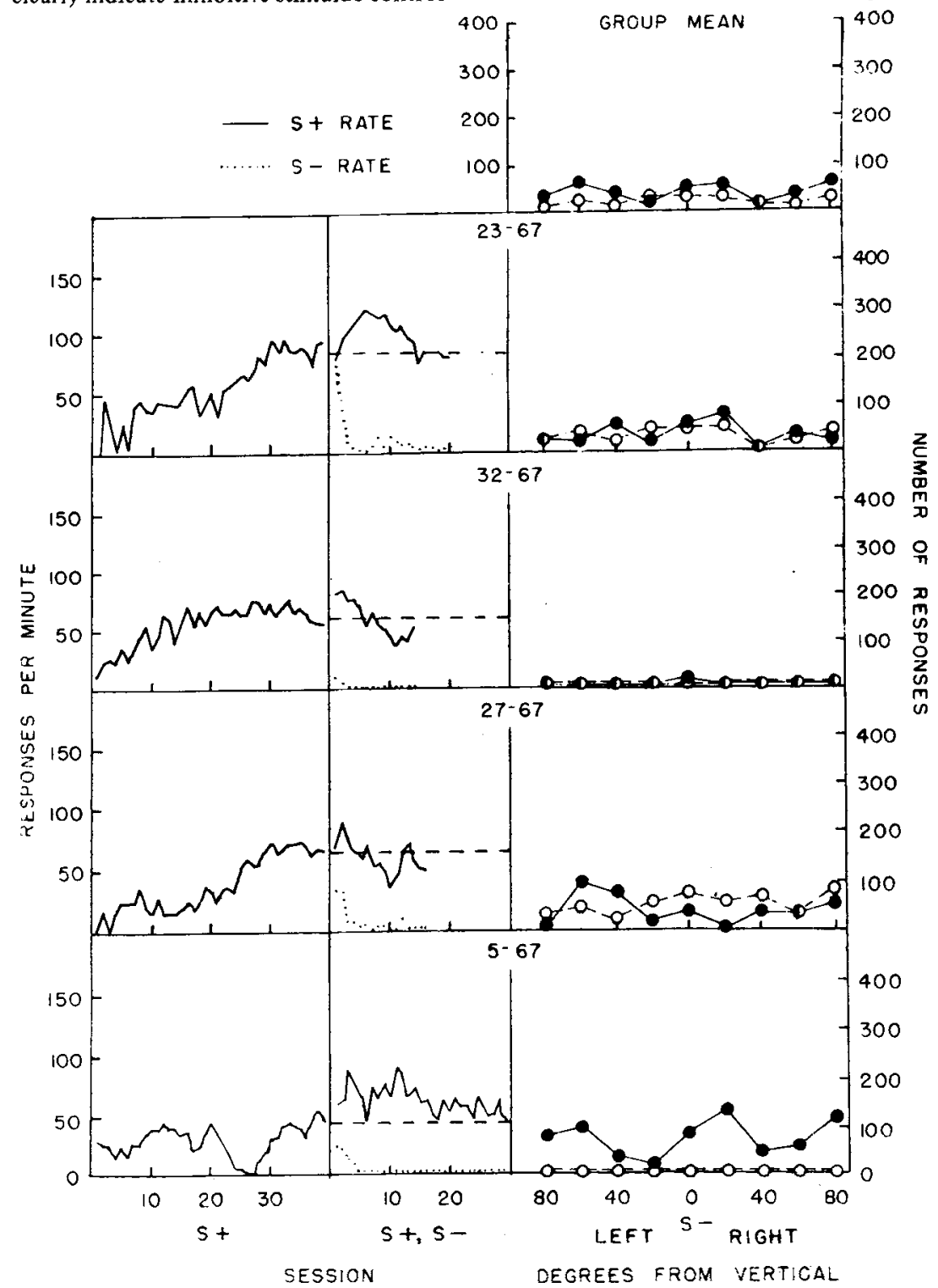

responding is low in the region of the $\mathbf{S}$ and increases with increasing distance from the $\mathrm{S}-$. In two cases, more responses were emitted when the background was black than when it was the same wavelength as the S+. The group mean data do not show any difference in mean number of responses between the two test conditions.

Figure 2 shows data of the birds tested when response rates during discriminative training were at the prediscriminative training level. None of the test data of the four birds indicate inhibitive stimulus control. These test data, like those in Fig. 1, do not show any difference in number of responses emitted under the two test conditions. There are two comparisons in the data of Figs. 1 and 2 worth noting. One is the difference in inhibitive stimulus control already noted,

Psychon. Sci., 1970, Vol. 18 (1) 
number of responses emitted in tests where the data reflected inhibitive stimulus control. In addition, it might be noted that pigeons that showed steep inhibitory gradients also had high rates of responding on the last day of discriminative training.

Finally, "superimposing" the S- on the $\mathrm{S}+$ dimension did not consistently increase the frequency of key pecks in the presence of the $\mathrm{S}$ - dimension in either of the two groups.

\section{DISCUSSION}

Pigeons tested when behavioral contrast was still in effect showed "inhibitive stimulus control" (a generalization gradient with a minimal number of key pecks in the region of $\mathrm{S}-$ ). Pigeons tested when behavioral contrast was no longer in effect did not show any evidence of inhibitive stimulus control.

Terrace (1966) has shown that when discriminative training is continued beyond the stage at which behavioral contrast is evident, rate of responding returns to the prediscriminative training level (i.e., behavioral contrast disappears). Another apparent effect of the continued training is the disappearance of the peak shift in the generalization gradient (stimulus associated with modal responding no longer shifted away from $\mathrm{S}+$ in a direction opposite $\mathrm{S}-$ ). Farthing et al (1968) have suggested that the progressive disappearance of the peak shift with continued training might be due in part to the "... increasing specificity of . inhibitory control by $S$ " [p. 751]. In that study, gradients of inhibitive effects became steeper with continued discriminative training. However, response rates in S+ also increased with increased discriminative training and may have been an important factor in the increased steepness of inhibitive stimulus control. One might suggest that discriminative training that results in greater and greater behavioral contrast also produces correlative increases in inhibitive control (Farthing et al, 1968), but that if training is continued and behavioral contrast disappears, then the peak shift disappears (Terrace, 1966), and the data of the present study would support the additional suggestion that inhibitive stimulus control also weakens, or disappears, rather than becoming steeper with increased discriminative training.

\section{REFERENCES}

FARTHING, G. W., \& HEARST, E. Generalization gradients of inhibition after different amounts of training. Journal of the Experimental Analysis of Behavior, 1968, 11, 743-752.

GELLERMANN, L. W. Chance orders of alternating stimuli in visual discrimination experiments. Journal of Genetic Psychology, 1933, 42, 206-208.

HONIG, W. K., BONEAU, C. A., BURSTEIN, K. R., \& PENNYPACKER, H. S. Positive and negative generalization gradients obtained after equivalent training conditions. Joumal of Comparative \& Physiological Psychology, 1963, 56, 111-116.

TERRACE, H. S. Behavioral contrast and the peak shift: Effects of extended discrimination training. Journal of the Experimental Analysis of Behavior, 1966, 9, 613-617. NOTE

1. This research was supported in part by USPHS Grant No. MH 08819 from the National Institutes of Health to the University of Maryland where the research was conducted. Preparation of this paper was aided by Grant No. NGR 39-018-002 from the National Aeronautics and Space Administration to Bryn Mawr College. Reprints may be obtained by writing to the Department of Psychology, Bryn Mawr College, Bryn Mawr, Penn. 19010. I wish to thank David Orme-Johnson for his, valuable suggestions and assistance in various phases of this research. I wish also to thank Dr. Harry Friedman for his assistance. lesions suffer from hypodipsia. Thus, the overall picture of the effect of medial hypothalamic lesions is of overeating, underdrinking, and an improper response to incentives. This syndrome might be due to an inadequate response to deprivation in the sense that when access to the restorative substance is not ad lib, the lesioned animal shows poorer performance of the corrective response than does the normal animal.

Another area of the brain that has been implicated in food and water regulation is the amygdaloid complex (Grossman \& Grossman, 1963). Amygdaloid complex lesioned animals have also been shown to respond inappropriately to changes in such reinforcement parameters as amount of reward (Pubols, 1966; Schwartzbaum, 1960). Thus, amygdaloid complex lesions may also lead to a "motivational deficit" resulting from an inadequate response to deprivation.

To evaluate this hypothesis, about 7 weeks after lesioning, animals were placed on water deprivation for a period of 1 week while water intakes were observed. If the two types of lesions do produce a deficit in response to deprivation, the lesioned animals should not show an increase in intake over days.

\section{METHOD}

The Ss consisted of 37 male albino (Holtzman) rats about 100 days of age at the time of surgery. Surgery was done under ether anesthesia with a preanesthetic dose of atropine sulfate. Bilateral lesions were produced through a stainless steel electrode, $0.25 \mathrm{~mm}$ in diam, completely insulated (Epoxylite) except for the tip. An anodal current of $2 \mathrm{~mA}$ for $10 \mathrm{sec}$ was used with a rectal cathode. The lesion coordinates (DeGroot, 1959) were 6.8 AP, $-4.0 \mathrm{DV}$, and 3.6 lateral for the amygdaloid group, and $7.0 \mathrm{AP},-2.8 \mathrm{DV}$, and 0.6 lateral for the hypothalamic group. The control group underwent surgery, but no electrode was lowered into the brain. Seven weeks after surgery, access to water was restricted to $1 / 2 \mathrm{~h}$ per day at the same time every day for the next 7 days. At all times, food (Purina Chow Meal) was ad lib. Body weights and water intakes were measured.

At the end of the experiment, all Ss were sacrificed and the brains were removed for histological examination. The 13 animals classified as medial amygdaloid complex lesioned were found to have bilateral destruction of the cortical, medial, and basomedial nuclei of the amygdala. The 10 classified as medial hypothalamic showed bilateral destruction of the ventromedial and dorsomedial nuclei of the hypothalamus with some destruction of the arcuate nucleus, the fornix, and the 